\title{
Therapeutic Effect of Myogenic Cells Modified to Express Neurotrophic Factors in a Rat Model of Sciatic Nerve Injury
}

\author{
Dadon- Nachum $\mathrm{M}^{1}$, Ben-Zur $\mathrm{T}^{1}$,Srugo $\mathrm{I}^{2}$, Shamir $\mathrm{H} \mathrm{M}^{2}$, Melamed $\mathrm{E}^{1}$, Yaffe $\mathrm{D}^{3}$, Offen $\mathrm{D}^{1}$
}

Sciatic nerve injury may cause neurological deficits, particularly muscle weakness. Previous studies have shown that administration of neurotrophic factors (NTFs), naturally occurring proteins that support the development and survival of neurons, partially protected the damaged motor neuron in the injured sciatic nerve. In the current study, we have examined whether the administration of various combinations of transfected muscle progenitor cells (MPCs) populations, each expressing a single NTF (BDNF, GDNF, IGF-1 or VEGF) or conditioned media of such culture are capable of rescuing motor neurons in culture or in vivo.

We have found that the mixture of conditioned media collected from cultured myogenic cells (MPCs- MIX ${ }^{+}$) alleviated the toxic effect of exposure of the motor neuron cell line NSC34 to hypoxic environment. Furthermore, NTFs secreting cells transplantation, protected motor neurons in a unilateral rat sciatic nerve injury model: One day after the crush, rats underwent transplantation at the lesion site with rat myogenic cells expressing one of the four NTFs; a mixture of cells expressing all four NTFs (MPCs- MIX ${ }^{+}$), MPCs-GFP or PBS. We found that in rats injected with MPCs- MIX $^{+}$the motor function was markedly preserved, compared to groups injected with cells secreting a single NTF, GFP or PBS. Transplantation of the MPCs- MIX ${ }^{+}$significantly inhibited the degeneration of the neuromuscular junctions and enhanced the survival of the myelinated motor axons. The injection of MPCs- $\mathrm{MIX}^{+}$preserved the compound muscle action potential (CMAP) as was demonstrated by motor nerve conduction studies. Our findings suggest that MPCs induced to secrete several NTFs can synergistically alleviate symptoms of sciatic nerve injury and perhaps other motor neuron disorders..

\section{Introduction}

Many studies have demonstrated that neurotrophic factors (NTFs) protect peripheral motor neurons and contribute to enhanced axons regeneration and functional recovery. ${ }^{[1-9]}$

Several studies showed that brain derived neurotrophic factor (BDNF) have a beneficial effect on peripheral nerve regeneration and myelination and on the maintenance of muscles' mass ${ }^{[10-14]}$

Additional NTFs such as glial derived neurotrophic factor (GDNF), insulin growth factor 1 (IGF-1) and vascular endothelial growth factor (VEGF) are known to be essential for the development and survival of the peripheral nervous system. These factors have been shown to improve motor neuron survival, preserve neuromuscular junctions (NMJs) and enhance motor function in animal models of peripheral nerve injuries. $[1,2,5,6,10-13,15]$ We have also shown that administration of bone marrow derived mesenchymal stem cells, that secret high levels of neurotrophic factors, improved motor function and nerve conduction in a rat sciatic nerve crush model. ${ }^{[16]}$
However, despite the promising results of restoration and recovery of the motor function in the animals models, clinical trials of systemic or intrathecal administration of recombinant NTFs to patients suffering from peripheral nerve injuries have failed to demonstrate significant efficacy. ${ }^{[17-18]}$

A potential solution to the challenge of NTFs delivery could be the transplantation of genetically modified myogenic cells, which secrete these factors, into the host muscle. In this case, the cells' own machinery is used to constantly produce higher concentration of growth factors, which could then be easily delivered to the motor neurons. Motor neurons can bind, internalize and retrogradely transport NTFs from muscles in a receptor-dependent manner. ${ }^{[3-4]}$

Muscle progenitor cells (MPCs) are of special interest as an excellent, easily accessible cell type, with wellcharacterized markers associated with the various differentiation stages. MPCs are also relatively simple to clone and manipulate in culture. Clonal analysis of newborn myogenic cells demonstrated stable retention during many cell generations in culture of a committed program of

\footnotetext{
${ }^{1}$ Felsenstein Medical Research Center and Sackler School of Medicine, Tel-Aviv University;

${ }^{2}$ Koret School of Veterinary Medicine, The Hebrew University of Jerusalem, Rehovot;

JSRM/Vol8 No.1, 2012; P21

${ }^{3}$ Department of Molecular Cell Biology, Weizmann Institute of Science, Rehovot, Israel.
} 


\section{RESEARCH ARTICLE}

self-renewal and a default for myogenic terminal differentiation. ${ }^{[19-21]}$

In the present study, we have used cell populations of the rat myogenic cell line L8, genetically modified to express and secrete BDNF, GDNF, IGF-1 or VEGF. Each population expressing a single NTF (MPCs- BDNF; MPCs- GDNF; MPCs- IGF-1; MPCs- VEGF respectively). Here, we demonstrated that transplantation of a mixture of these genetically manipulated MPCs have strong synergistic effect in a rat model of sciatic nerve crush, the most commonly studied nerve injury paradigm. ${ }^{[22]}$ The MPC mixture harboring together the four NTF, accelerated the recovery of the motor function, preserved the compound muscle action potential (CMAP) and inhibited the degeneration of the neuromuscular junctions.

\section{Materials and Methods}

\section{Myogenic cells secreting neurotrophic factors}

L8 rat myogenic cells ${ }^{[21]}$ were used as a vehicle to introduce vectors expressing growth factors. The cells were propagated in a growth medium containing Dulbecco's Modified Eagle's Medium (DMEM) supplemented with 100 $\mathrm{mg} / \mathrm{ml}$ streptomycin, $100 \mathrm{U} / \mathrm{ml}$ penicillin, 12.5 units $/ \mathrm{ml}$ nystatin (SPN, Biological Industries, Beit Haemek, Israel), 2 $\mathrm{mML}$ - glutamine (Biological Industries), and $10 \%$ fetal calf serum (Biological Industries).

Following a change to differentiation medium the mononucleated cells ceased proliferation and fused into multinucleated muscle fibers. For the present investigation we used only proliferating mononuclated cells grown in the growth medium.

The human GDNF, VEGF, IGF-1 and BDNF genes were amplified from the pBluescript plasmids that were purchased from Harvard Institute of Proteomics, Boston, USA, using Plasmid Midi Kit (Qiagen, Valencia, USA). We inserted each of the four genes into the destination plasmid under the CMV promoter in a recombinant reaction. The destination vector is a commercial expression vector (Invitrogen Cat. K591-20) in to which the promoter and gene of interest are simultaneously cloned according to manufacturer's instructions. The vector also contains the Blasticidin resistance marker to select the cells expressing the vector. For each reaction sample, a plasmid containing the CMV promoter was incubated overnight at room temperature with a plasmid containing the DNA fragment, the destination plasmid and the recombination enzyme, LR clonase (Invitrogen Carlsbad, CA, USA). The constructs were transformed into One Shot ${ }^{\circledR}$ Stbl $3^{\mathrm{TM}}$ Competent E. coli (Invitrogen): $4 \mu \mathrm{l}$ of the recombination reaction were added to One Shot ${ }^{\circledR}$ Stbl $3^{\mathrm{TM}}$ Competent E. coli and were incubated for 30 minutes on ice. After incubation, the mix was transferred to $42^{\circ} \mathrm{C}$ for 30 seconds and then for 2 minutes on ice. $250 \mu \mathrm{l}$ of SOC medium was added to the mix and incubated at $37^{\circ} \mathrm{C}$. After one hour the mix was placed on LB agar plates with ampicillin (Sigma- Aldrich, St. Louis, MO, USA) for 24 hours at $37^{\circ} \mathrm{C}$. On the following day, one colony was picked and DNA was produced using a midi kit. For each transfection sample, $4 \mu \mathrm{g}$ DNA (of each gene separately) were diluted in $1.5 \mathrm{ml}$ Opti- MEM (Biological Industries). Lipofectamaine 2000 (Invitrogen) was diluted in $1.5 \mathrm{ml}$ Opti-MEM. After 5 minutes of room temperature incubation, the diluted mix and the DNA with the diluted Lipofectamine 2000 were combined and incubated for 20 minutes at room temperature. After incubation, the $3 \mathrm{ml}$ of complexes were added to flasks containing 95\% confluent MPCs cultured in an antibiotic free medium and incubated at $37^{\circ} \mathrm{C}$ in a $\mathrm{CO}_{2}$ incubator. Each flask was transfected with a single NTF expression vector. Six hours later, the cell medium was changed to their standard growth medium. The next day, $4 \mathrm{mg} / \mathrm{ml}$ of Blastocidin was added to the cells for selection after two weeks.

\section{Immunocytochemistry}

Cells grown on coverslips were fixed with $4 \%$ paraformaldehyde for 10 minutes, washed with phosphatebuffered saline (PBS), and then incubated in a blocking and permeabilization solution ( $5 \%$ normal goat serum, $1 \%$ bovine serum albumin, and $0.5 \%$ Triton $\mathrm{X}-100$ in PBS) and incubated with a primary antibody overnight at $4^{\circ} \mathrm{C}$. After washing with PBS, cells were incubated with an Alexaconjugated secondary antibody. The nuclei were counterstained with 4,6-diamidino-2-phenylindole (DAPI; 1:500; Sigma-Aldrich). The following primary antibodies were used: rabbit a-BDNF (1:100; Santa Cruz Biotechnology Inc., Santa Cruz, CA, USA), rabbit $\alpha-$ GDNF ( 1:100; Santa Cruz Biotechnology), mouse $\alpha-$ IGF-1 (1:100; Santa Cruz Biotechnology), and mouse $\alpha-$ VEGF (1:100; Santa Cruz Biotechnology). Secondary antibodies were Alexa Fluor 488 (1:500; Invitrogen) and Alexa Fluor 568 (1:500; Invitrogen). Five random fields were photographed at a magnification of $\mathrm{X} 100 \mathrm{~A}$ and a percentage of total DAPI-positive nuclei cells were quantified.

\section{ELISA analysis}

Cell supernatant collected pre- and post- transfection were, frozen, and quantified. We used enzyme-linked immunosorbent assay (ELISA) kits (R\&D Systems, Minneapolis, MN, USA) to detect the presence of each one of the secreted neurotrophic factors. The assay was conducted according to the manufacturer's protocol in triplicate, and results were read at wavelengths of $450 / 540$ using an ELISA reader (Powerwave X; Biotek Instruments, Winooski, VT, USA). Results were compared between the cell media prior to and following transfection.

\section{Cells viability assay}

We have tested the ability of the MPCs conditioned media to protect motor neuron cell- line (NSCs-34) from hypoxic stress. NSCs-34 was placed in a hypoxic environment for 48 hours in a chamber together with the conditioned media of each of the cloned MPCs expressing one of the four NTF combinations: MPCs-MIX ${ }^{+}$MPCs-GFP, MPCs growth media media or serum free growth media. After 48 hours, Alamar blue $10 \%$ (AbD Serotec, Kidlington, UK) was added to the cells for 6 hours. The assay was conducted in triplicate and results were read at wavelengths of $590 \mathrm{~nm}$ using a fluostar device. Results were normalized to cells under the same treatments in normoxia. 


\section{RESEARCH ARTICLE}

\section{$\underline{\text { Sciatic nerve crush model in rats }}$}

The sciatic nerve crush model was performed on male Wistar rats (Harlan, Jerusalem) weighing 230-250 g each. Rats were placed in 12-hour-light/12-hour-dark conditions and were grown in individually ventilated cages (IVC) with $\mathrm{ad}$ libitum access to food and water. All experimental protocols were approved by the Tel Aviv University Committee of Animal Use for Research and Education. Rats were anesthetized with chloral hydrate $7 \mathrm{mg} / \mathrm{ml}$ (Sigma- Aldrich, St. Louis, USA). The right sciatic nerve was exposed and a vessel clamp was applied $10 \mathrm{~mm}$ above the first branching of the nerve, for 30 seconds.

One day after surgery, the rat groups $(n=9)$ were injected into the lesion site with the genetically modified cells suspended in $100 \mu \mathrm{L}$. The groups included rat that were transplanted with $10^{6}$ MPC harboring the gene for green fluorescent protein (GFP), $10^{6}$ MPC harboring the gene for one of the NTFs, i.e.: BDNF, GDNF, IGF-1 or VEGF. One group was transplanted with a mixture of cells expressing all these NTF genes (i.e. $2.5 \times 10^{5} \times 4$ a total of $10^{6}$ cells) and a rat group that was injected with PBS alone.

\section{Rat motor function measurements}

Motor function was evaluated twice a week- a week before the injury and three weeks after. Motor activity was measured using the San Diego Instrument test (San Diego Instruments, CA, USA). This test measured the time that the rat remains balanced on a rotating rod in accelerated speed (0-25 RPM). Following a brief training period, adult wild-type rats are able to remain balanced for up to 4 minutes. Sciatic nerve crush, as was done to the rats in this study, damages the rat's ability to balance, hence causing the animal to fall off the rod after shorter periods of time. The average time measured in three consecutive runs for each rat was recorded and the groups' performance was compared.

\section{Electrophysiological study}

Compound muscle action potentials (CMAP) were recorded from the sciatic innervated cranial tibial muscles following electric stimulation of the sciatic nerve. An active monopolar needle electrode was placed over the sciatic nerve at the sciatic notch and a supramaximal intensity electric stimulus of $0.1 \mathrm{~ms}$ duration was applied. An average of ten consecutive runs from each measurement was documented. The CMAP amplitudes were recorded on both the injured and the healthy hind limbs of each rat. The ratios of the amplitude measured at the injured side, divided by those of the normal side was used for comparison between the study group in order to adjust for the effect of anesthesia, muscle masses and other physical variations between rats.

\section{Immunohistochemistry}

To analyze cell survival and the NTFs secretion in the tissue, hind limb muscles of rats were removed and frozen in liquid nitrogen, 15 days after transplantation. Muscles were sectioned at $20 \mu \mathrm{m}$ using a cryostat (Leica CM1850) and placed on glass slides for staining. The sections were fixed with $4 \%$ paraformaldehyde for 30 minutes, washed with phosphate-buffered saline (PBS), and then incubated in a blocking and permeabilization solution (5\% normal goat serum, $1 \%$ bovine serum albumin, and $0.5 \%$ Triton $X-100$ in PBS) and incubated with a primary antibody overnight at $4^{\circ} \mathrm{C}$. After being washed with PBS, cells were incubated with an Alexa-conjugated secondary antibody. The nuclei were counterstained with 4, 6-diamidino-2-phenylindole (DAPI; 1:500; Sigma-Aldrich). The following primary antibodies were used: rabbit $\alpha-B D N F$ (1:100; Santa Cruz Biotechnology Inc., Santa Cruz, CA, USA), rabbit $\alpha-$ GDNF (1:100; Santa Cruz Biotechnology), mouse $\alpha-$ IGF-1 (1:100; Santa Cruz Biotechnology), and mouse $\alpha-$ VEGF (1:100; Santa Cruz Biotechnology).Secondary antibodies were Alexa Fluor 488 (1:500; Invitrogen) and Alexa Fluor 568 (1:500; Invitrogen).

\section{Assessment of neuromuscular junction innervations}

Endplate innervations were marked by alpha-bungarotoxin and synaptophysin as described previously. Hind limb muscles were dissected and frozen in liquid nitrogen. Muscles were sectioned at $20 \mu \mathrm{m}$ using a cryostat and placed on glass slides for staining. The sections were fixed with 4\% PFA-PBS and labeled with alpha-bungarotoxin conjugated with fluorescence marker Alexa Fluor 594 (1:1000, Invitrogen, CA, USA) and anti-synaptophysin (rabbit polyclonal, 1:100, Santa Cruz Biotechnology, Santa Cruz, USA) antibodies overnight at $4^{\circ} \mathrm{C}$. After washing with PBS, the sections were incubated with anti- rabbit Alexa Fluor 488conjugated antibody (1:1,000, Invitrogen) for one hour at room temperature followed by washes and covered with cover glasses using aqueous mounting medium (Invitrogen). We classified NMJs into two groups based on the degree of innervation of postsynaptic receptor plaques by nerve terminals. Endplates were scored as "innervated" if there was an overlap with the axon terminal, or "denervated" if the endplate was not associated with an axon.

\section{Statistical Analysis}

The results are expressed as means \pm SE. The one-way ANOVA test was used to compare the three groups in the sciatic nerve crush and SOD1 mice models. Statistical calculations were performed using SPSS, version 13 (SPSS, Chicago, IL, USA).

\section{$\underline{\text { Results }}$}

\section{Characterization of the transfected myogenic cells}

Using an immunocytochemical and ELISA analyses, we assessed the expression and secretion of neurotrophic factors from MPC-transfected cells. We found that almost $100 \%$ of the genetically manipulated MPCs strongly expressed BDNF, GDNF, IGF-1, or VEGF. We found, per one million cells, high levels of BDNF $(949.6 \pm 53 \mathrm{pg})$, GDNF $(900.5 \pm 42 \mathrm{pg})$, IGF-1 $(989.7 \pm 63 \mathrm{pg})$ and $\operatorname{VEGF}(879.1 \pm$ $146 \mathrm{pg})$. In contrast, we couldn't detect any NTF secretion in untransfected MPCs (Figure 1). 

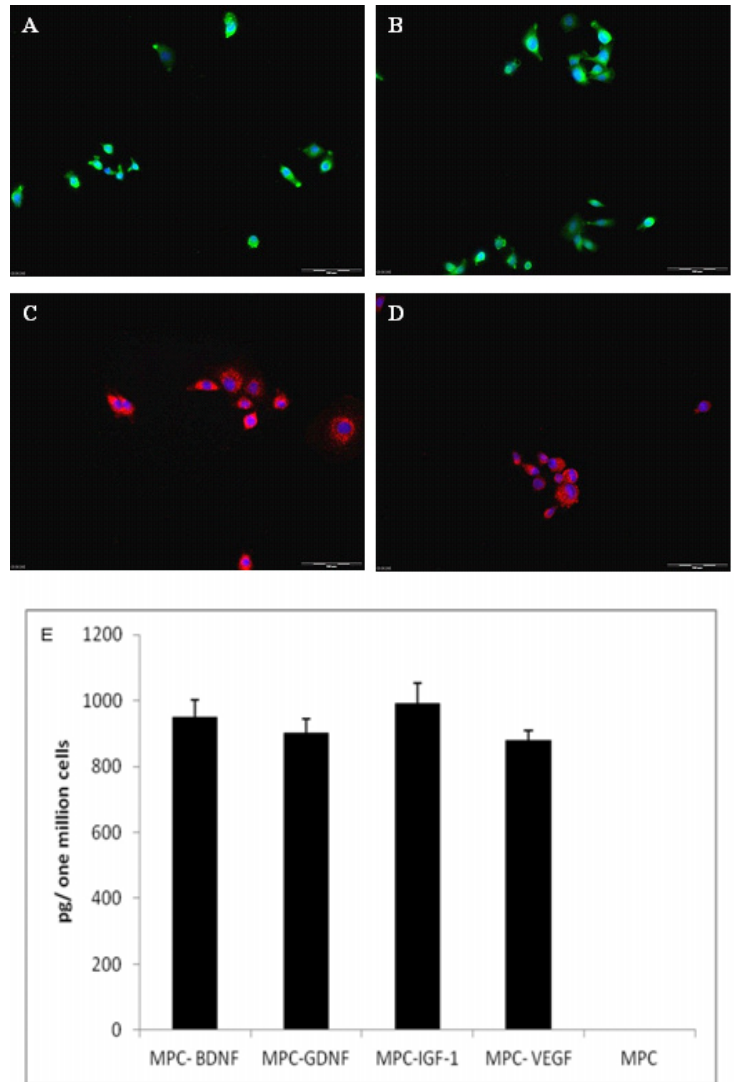

Figure 1 (a to e) Genetically modified myogenic cells secrete neurotrophic factors. L8 cells transfected with the neurotrophic factors genes, express BDNF (A) GDNF (B) IGF-1 (C) and VEGF (D) as determined by immunocytochemistry. These factors were secreted into the medium as indicated by ELISA (E). No secreted NTFs were detected in the nontransfected L8 cells (MPCs).

\section{MPCs-MIX ${ }^{+}$conditioned media rescue NSC34 cells in culture}

48 hours after exposure to a hypoxic environment, the viability of the motor neuron cell line NSCs-34 decreased by $50 \%$. Exposure of the cells to conditioned medium collected from cells expressing only a single NTF (BDNF, GDNF, IGF1 or VEGF) had an insignificant protective effect $(64.3 \% \pm$ $3.3,66.8 \% \pm 1.9,66.03 \% \pm 3.7$ and $63.4 \% \pm 2 . \overline{9}$ respectively). However, cells treated with the MPCs-M $\bar{M} \mathrm{X}^{+}$ conditioned medium were almost fully protected $(97.4 \% \pm$ 7.3; Figure 2).

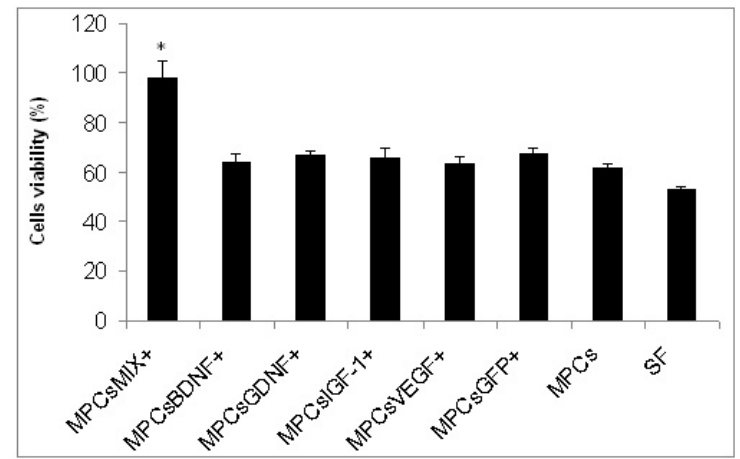

Figure 2. Conditioned medium of genetically modified L8 cells (MPCs $\mathrm{MIX+)}$ rescues NSC34 cells in culture. NSC34 cells were exposed to hypoxic environment for 48 hours in serum free medium (SF) or in SF and conditioned media collected from cells expressing only a single NTF or in a mixture of medium collected from MPCs expressing each one of the four NTFs ( ${ }^{*} \mathrm{P}<0.05$ as determined by ANOVA test).
Transplantation of genetically modified L8 cells expressing NTFs improved motor function

Rats that performed well on the rotorod before the injury, demonstrated right hind limb limp immediately following the nerve crush and their motor function declined by $60 \%$.

Three days later, the PBS-treated rat group demonstrated poor motor function $(98.5+9.8$ seconds on rotorod). Injured rats treated with cells expressing GFP or cells expressing a single NTF (i.e.: BDNF, GDNF, IGF-1 or VEGF) showed an insignificant, moderate improvement $(125 \pm 12.5 ; 152 \pm 9.7$; $129 \pm 11.2 ; 129 \pm 13.4 ; 121 \pm 9.7$, respectively). In contrast, the ${ }^{-}$MPCs-MIX ${ }^{+}$cells treated rats demonstrated a significantly improved motor performance $(183.8 \pm 5.3, p<$ 0.05). A similar trend was observed six and eight days after the crush (Figure 3).

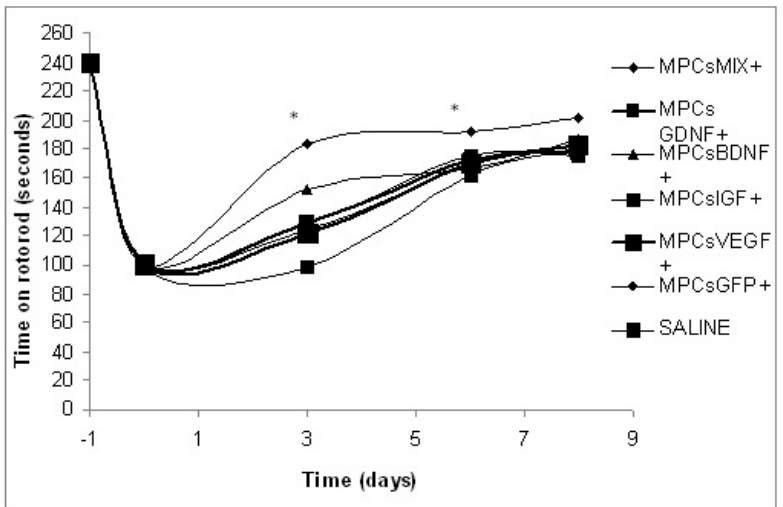

Figure 3. Transplantation of genetically modified L8 cells expressing NTFs improved motor function after sciatic nerve injury. One day after a crush of the rats' right hind limb, myogenic cells expressing one of the four NTFs or GFP or a mixture of myogenic cells expressing all four NTFs were transplanted into the injury site. One group was injected with saline only. Motor recovery was examined by rotarod and presented as time spent on rod. In rats injected with MPCs-MIX, motor function was markedly improved, compared to the other treatments. ( $n=9,{ }^{*} p<0.05$ as determined by ANOVA test).

Decreased compound muscle action potential (CMAP) was observed immediately after the sciatic crush in the injured limb in all groups when compared to the non-injured contra lateral side. These findings were recorded as low CMAP amplitude ratios (injured/healthy limb). Four days after

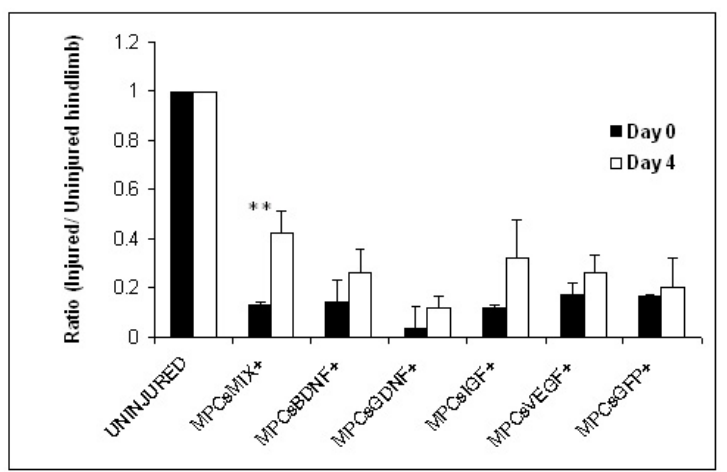

Figure 4. Electrophysiologic measurement indicates enhanced axonal regeneration in MPCs-MIX treated rats. Myogenic cells expressing one of the four NTFs or GFP or a mixture of myogenic cells expressing all four NTFs, or PBS were injected into sciatic nerve crush site. Four days later, nerve conduction was tested by electromyography. Compound muscle action potential presented as a ratio between the injured and uninjured hind limbs. A significant enhanced axonal regeneration was observed only in the MPCs- MIX ${ }^{+}$treated group. $\left(n=3\right.$, means \pm SEM, ${ }^{* *} p<0.01$ as determined by student t- test) 


\section{RESEARCH A R T I C L E}

surgery, the average ratio of CMAP amplitudes (injured/healthy limb) was higher in the MPCs- GFP, BDNF, GDNF, IGF-1 or VEGF $(0.2 \pm 0.12 ; 0.26 \pm 0.09 ; 0.12 \pm 0.04$; $0.32 \pm 0.16 ; 0.26 \pm 0.07$, respectively) when compared to the ratios recorded immediately after the surgery. However, a significant increase in the CMAP amplitudes ratio was found only in the MPCs-MIX ${ }^{+}$treated group $(0.4 \pm 0.09 p<0.05$, Figure 4).

\section{Myogenic cell populations that secrete NTFs inhibited NMJs denervation}

Two weeks after the sciatic nerve crush, intact NMJs were counted within the gastrocnemius and tibialis muscles in the crushed area. Positive staining with acetylcholine receptor ligand, alpha bungarotoxin, and antibodies against the post Synaptic protein, synaptophysin, were count as innervated NMJ. We examined over 50 stained slides from each group, and found that in the injured gastrocnemius and tibialis muscles $69 \% \pm 12$ innervated NMJs. In contrast, rats transplanted with MPC-MIX cells showed significantly higher intact NMJ preservation $(89 \% \pm 1.5, p<0.05$; Figure 5).
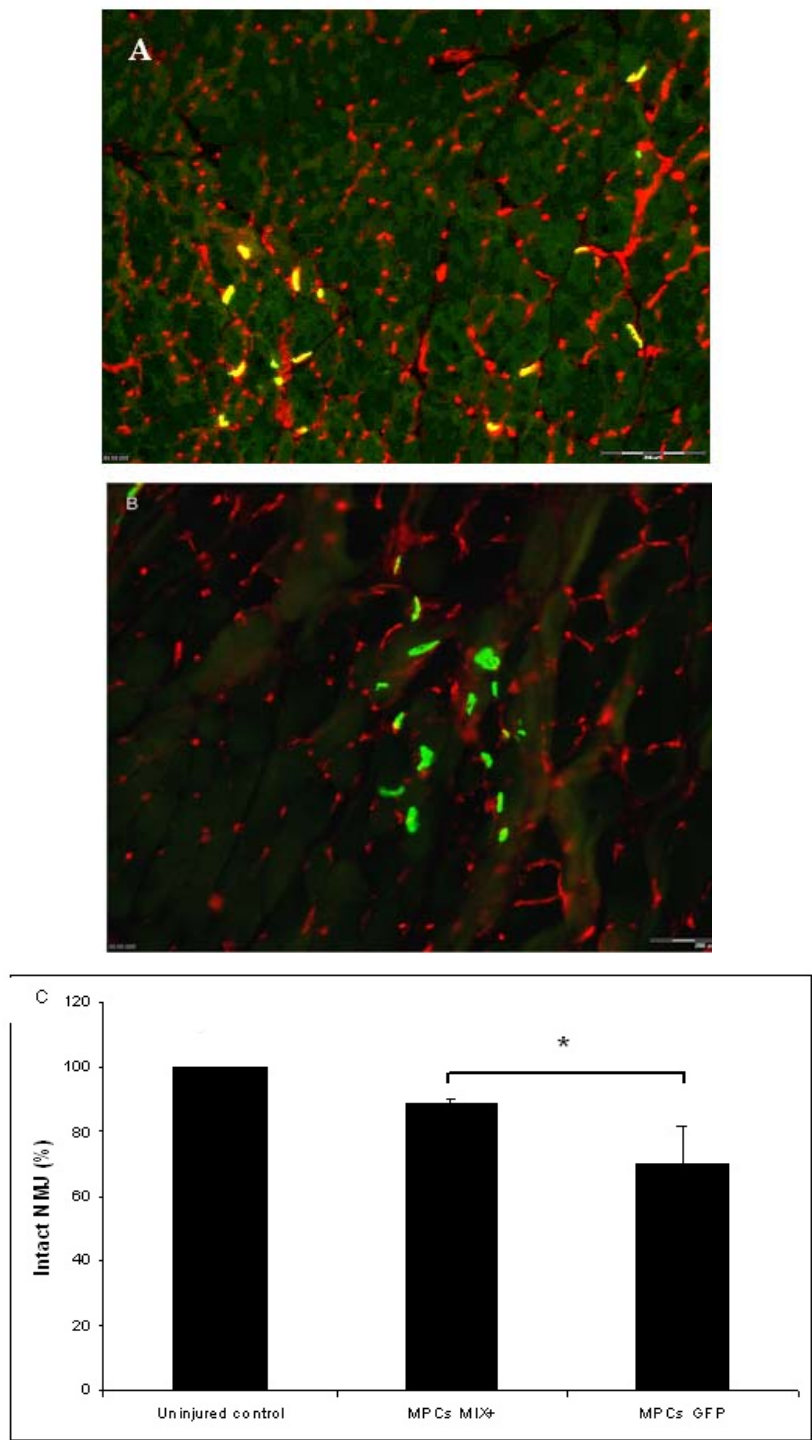

Figure 5. Injected myogenic cells that secrete NTFs reduced NMJs denervation. Two weeks after sciatic nerve crush and transplantation of myogenic cells expressing all four NTFs, hind limbs muscles were double stained using alpha bungarotoxin (green) and antisynaptophysin antibodies (red) to measure MNJ integrity. A- Image of hind limb muscle section transplanted with MPCs-MIX $(100 \mu \mathrm{m})$ B- or with MPCs- GFP+. CQuantification of intact NMJs $\left(n=5\right.$, means + SEM, ${ }^{*} p<0.05$, determined by student t-test).
Cells expressing NTFs can be detected in the transplanted muscles

Cells expressing high levels of BDNF, GDNF, IGF-1 or VEGF were identified on histology examination of the rat's hind limb muscles two weeks after the transplantation. Immunohistochemistry using specific antibodies was used to detect these factors (Figure 6).
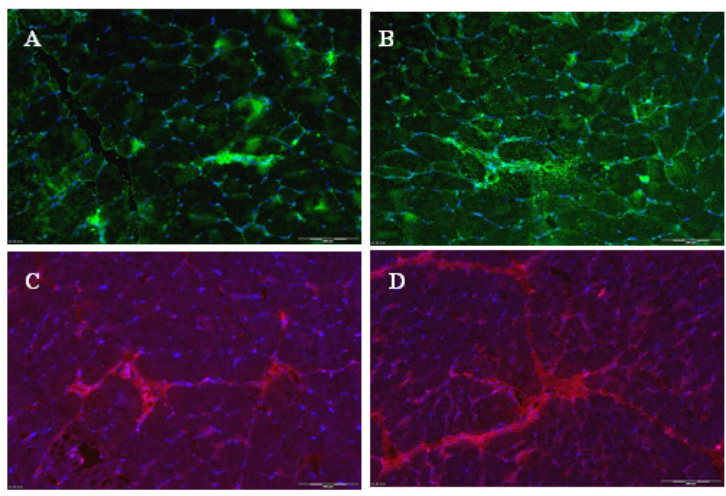

Figure 6. Cells expressing NTFs can be detected in the transplanted muscles. Rats were sacrificed two weeks after sciatic nerve crush followed by transplantation of a mixture of myogenic cells expressing a mixture of myogenic cells that express together all four NTFs (MPCs MIX+). Cells in hind limb muscles sections were stained positive for BDNF (A); GDNF (B); IGF-1 (C) and VEGF (D).

\section{Discussion}

Many studies have reported positive effects of different NTFs in models of motor neurons pathologies, ${ }^{[23-26]}$ but only few have tested the possible synergistic effect of their combination. ${ }^{[27]}$ It is very likely that each NTF has a unique important role in the survival and maintenance of the motor neurons. Therefore, it is reasonable to explore their combined effect. In the present study, using several parameters, we found that both in-vitro and in-vivo administration of all four NTFs has a beneficial synergistic effect in a model of acute motor neuron injury.

Sciatic nerve crush is a very common peripheral nerve injury. Although this injury is widely investigated, no efficient treatment has yet been found. Since the nerve axon usually regenerates slowly (1-2 $\mathrm{mm} /$ day) muscle regeneration is hampered. Chronic muscle atrophy and fibrosis limit the recovery, and compromise the restoration of muscle function. ${ }^{15,28-31]}$ Enhanced nerve repair and prevention of muscle atrophy represent major challenges for restorative medicine.

One of the suggested treatment strategies in peripheral nerve injuries is the transplantation of stem or progenitor cells in order to create a better environment for nerve regeneration. Among others, adipose precursor cells, amniotic fluid cells and bone marrow mesenchymal stem cells were tested. ${ }^{[15,16,24-26,29,32-34]}$ One of the postulated mechanisms for this beneficial effect was the secretion of NTFs. NTFs are known as regulators of some of the important processes of the nervous system, including neuronal survival, axonal growth and branching, synaptic efficacy etc. However, most of these implanted cells have a short survival time and thus their efficacy is limited. ${ }^{[25-26]}$

In the present study, we genetically modified four separated cloned populations of myogenic cells, each population expressina one of the followina neurotrophic factors aenes: 


\section{RESEARCH ARTICLE}

BDNF, GDNF, IGF-1 or VEGF (MPCs-NTFs). Our experiments offer a fairly simple way to test the combined effect by mixing defined numbers of cells expressing the different NTFs. We showed that only the combined conditioned medium from all four populations significantly rescued a cultured motor neurons cell line from hypoxic damage. Subsequently, we applied these NTFs secreting cells in the rat model of unilateral sciatic nerve crush. We used several parameters to analyze the regenerated motor axons and the integrity of the hind limb NMJs following sciatic nerve crush. One day after injury, we observed a marked decrease in motor function as indicated by the rotorod test and CMAP measurements. MPCs were transplanted into the lesion site $24 \mathrm{~h}$ after injury. We observed a significant beneficial effect on the motor function in the rats transplanted with MPCs-MIX, as soon as four days after the treatment. The high CMAP amplitude recorded in the hind limb muscles of rats transplanted with a mix of MPC expressing the four NTFs indicated that the transplantation preserved the myelinated motor axons and innervated peripheral muscles. In addition, we observed a marked reduction in degeneration of the NMJs in the rats transplanted with MPCs-MIX .

Several attempts to introduce and evaluate the effect of NTFs on an injured nerve environment were reported. Li et al. (2007) showed that overexpression of GDNF in the skeletal muscle delayed the onset of disease and increased the life span of SOD1 mice. Acsadi et al. (2002) showed that adenovirus vector mediated GDNF expression in muscles of neonatal SOD1 mice delayed the onset of disease and prolonged animals' survival. Kaspar et al. (2003) reported that adenovirus vector mediated IGF-1 expression in SOD1 mice muscles prolonged life and delayed disease progression. Azzouz et al. (2004) found that injection of a VEGF-expressing lentiviral vector into various muscles delayed onset and slowed progression of ALS in mice..$^{[1,4,23,}$ $35]$

Our results, from the current study, demonstrate that NTFs synergy is very important for motor neurons regeneration after injury. Although introducing each of these factors alone resulted in some beneficial effect, the effect of the combination of all factors together was significantly superior.

\section{Conclusion}

Muscle is an accessible tissue and primary MPCs can be isolated in sufficient amounts making these adult progenitor cells potentially clinically feasible for cell transplantation therapies. In our study, the transplanted cells survived, expressed and secreted BDNF, GDNF, IGF-1 and VEGF in the injured tissue throughout the entire experiment period, three weeks after transplantation. The results from this study demonstrate that the NTFs secreted from the myogenic cells have a synergistic protective effect in supplying a nurturing environment to the injured nerve. Moreover, our study suggests that transplantation of genetically modified myogenic cells might be used in the future for autologous transplantation after peripheral nerve injury.

\section{Acknowledgments}

The authors wish to thank Dr. Igor Tarasenko for his exceptional assistance with the animal model and to Ms. Danielle Sabah-Israel for her editorial assistance.

\section{References}

1. Azzouz M, Ralph GS, Storkebaum E, Walmsley LE, Mitrophanous KA, Kingsman SM, Carmeliet P, Mazarakis ND. VEGF delivery with retrogradely transported lentivector prolongs survival in a mouse ALS model. Nature. 2004; 429 (6990): 413-7.

2. Crone SA, Lee KF. Gene targeting reveals multiple essential functions of the neuregulin signaling system during development of the neuroendocrine and nervous systems. Ann. N. Y. Acad. Sci.2002; 971: 547-53.

3. Dobrowolny G, Giacinti C, Pelosi L, Nicoletti C, Winn N, Barberi L, Molinaro M, Rosenthal N, Musaro A. Muscle expression of a local Igf- 1 isoform protects motor neurons in an ALS mouse model. J. cell Biol. 2005;168(2): 193-9.

4. Li W, Brakefield D, Pan Y, Hunter D, Myckatyn TM, sParsadanian A. Muscle-derived but not centrally derived transgene GDNF is neuroprotective in G93A-SOD1 mouse model of ALS. Exp. Neurol. 2007; 203(2): 457-71.

5. Musarò A, McCullagh K, Paul A, Houghton L, Dobrowolny G, Molinaro M, Barton ER, Sweeney HL, Rosenthal N. Localized Igf-1 transgene expression sustains hypertrophy and regeneration in senescent skeletal muscle. Nat. Geneti. 2001; 27(2): 195-200.

6. Rabinovsky ED, Gelir E, Gelir S, Lui H, Kattash M, DeMayo FJ, Shenaq SM, Scheartz RJ. Targeted expression of IGF-1 transgene to skeletal muscle accelerates muscle and motor neuron regeneration. FASEB J. 2003; 17(1):53-5.

7. Storkebaum E, Lambrechts D, Dewerchin M, MorenoMurciano MP, Appelmans S, Dh H, Van Damme P, Rutten B, Man WY, De Mol M, Wyns S, Manka D, Vermeulen K, Van Den Bosch L, Mertens N, Schmitz C, Robberecht W, Conway EM, Collen D, Moons L, Carmeliet P. Treatment of motoneuron degeneration by intracerebroventricular delivery of VEGF in a rat model of ALS. Nat. Neurosci. 2005; 8(1): 85-92.

8. Wang Y, Mao XO, Xie L, Banwait S, Marti HH, Greenberg DA, Jin K. Vascular endothelial growth factor overexpression delays neurodegeneration and prolongs survival in amyotrophic lateral sclerosis mice. J. Neurosci. 2007; 27(2): 304-7.

9. Zheng C, Sköld MK, Li J, Nennesmo I, Fadeel B, Henter JI. VEGF reduces astrogliosis and preserves neuromuscular junctions in ALS transgenic mice. Biochem. Biophys. Res. Commun. 2007; 363(4): 989-93.

10. Fryer HJ, Wolf DH, Knox RJ, Strittmatter SM, Pennica D, O'Leary RM, Russell DS, Kalb RG. Brain-derived neurotrophic factor induces excitotoxic sensitivity in cultured embryonic rat spinal motor neurons through activation of the phosphatidylinositol 3-kinase pathway. J. Neurochem. 2000; 74 (2): 582-95.

11. Hu P, Kalb RG. BDNF heightens the sensitivity of motor neurons to excitotoxic insults through activation of TrkB. J. Neurochem. 2003; 84(6): 1421-30.

12. Mousavi K, Parry DJ, Jasmin BJ. BDNF rescues myosin heavy chain IIB muscle fibers after neonatal nerve injury. Am. J. Physiol. 2004; 287(1): C22-9. 
13. Ozdinler PH, Macklis JD. IGF-I specifically enhances axon outgrowth of corticospinal motor neurons. Nat. Neurosci. 2006; 9(11): 1371-81.

14. Zhang J, Luo X, Xian C, Liu Z, Zhou X. BDNF is required for myelination and regeneration of injured sciatic nerve in rodents. Eur. J. Neurosci. 2000;12(12):4171-4180.

15. Goel R K, Suri V, Suri A, Sarkar, Mohanty S, Sharma MC, Yadav PK, Srivastava A. Effect of bone marrow-derived mononuclear cells on nerve regeneration in the transection model of the rat sciatic nerve. J. Clin. Neurosci. 2009; 16(9), 1211- 1217.

16. Dadon- Nachum M, Sadan O, Srugo I, Melamed E, Offen D. Differentiated mesenchymal stem cells for sciatic nerve injury. Stem Cell Rev. 2011; Epub ahead of print.

17. Miller RG, Petajan JH, Bryan WW, Armon C, Barohn RJ, Goodpasture JC, Hoagland RJ, Parry GJ, Ross MA, Stromatt SC A placebo- controlled trial of recombinant human ciliary neurotrophic (rhCNTF) factor in amyotrophic lateral sclerosis. rhCNTF ALS study group. Ann. Neurol. 1996; 39 (2): 256-60.

18. Stender M. Gene therapy for motor neuron disease. Nat. Med.1997; 3 (4); 380-1.

19. Yaffe D. Cellular aspects of muscle differentiation in vitro. Curr. Top. Dev. Biol. 1969; 4: 37- 77.

20. Yaffe D, Saxel O. Serial passaging and differentiation of myogenic cells isolated from dystrophic mouse muscle. Nature, 1977; 270 (5639): 725- 7.

21. Yaffe D, Saxel O. A myogenic cell line with altered serum requirements for differentiation. Differentiation,1977; 7:159-66.

22. Konstantinou K, Dunn KM. Sciatica: review of epidemiological studies and prevalence estimates. Spine, 2008; 33 (22):2464-2472.

23. 23. Acsadi G, Anguelov R, Yang H, Toth G, Thomas $R$, Jani A, Wang $Y$, lanakova $E$, Mohammad S, Lewis RA, Shy ME. Increased survival and function of SOD1 mice after Glial cell- derived neurotrophic factor gene therapy. Hum. Gene Ther. 2002; 10 (13):1047-1059.

24. Mohajeri H, Figlewicz D, Bohn M. Intramuscular grafts of myoblasts genetically modified to secrete glial cell line- derived neurotrophic factor prevent motoneuron loss and disease progression in a mouse model of familial amyotrophic lateral sclerosis. Hum. Gene Ther.1999; 10: 1853- 1866.

25. Pan HC, Yang DY, Chiu YT, Lai SZ, Wang YC, Chang $\mathrm{MH}$, Cheng FC. Enhanced regeneration in injured sciatic nerve by human amniotic mesenchymal stem cells. J. Clin. Neurosci. 2006; 13: 570-575

26. Pan HC, Cheng FC, Chen CJ, Lai SZ, Lee CW, Yang DY, Chang $\mathrm{MH}$, Ho SP. Post injury regeneration in rat sciatic nerve facilitated by neurotrophic factors secreted by amniotic fluid mesenchymal stem cells. J. Clin. Neurosci. 2007; 14: 1089-1098.

27. Logan A, Ahmed Z, Baird A, Gonzalez AM, Berry M. Neurotrophic factor synergy is required for neuronal survival and disinhibited axon regeneration after CNS injury. Brain, 2006; 129: 490-502.

28. Fawcett JW, Keynes RJ. Peripheral nerve regeneration. Annu. Rev. Neurosci. 1990; 13:43-60.

29. Gu S, Shen Y, Xu W, Xu L, Li X, Zhou G, Gu Y, Xu J . Application of fetal neural stem cells transplantation in delaying denervated muscle atrophy in rats with peripheral nerve injury. Microsurgury, 2010; 30(4); 266-74

30. Hall, S. (2001). Nerve repair: a neurobiologist's view. Journal of Hand Surgery, 26:129-36.

31. Rochkind S, Geuna S, Shainberg A. Phototherapy in peripheral nerve injury: effects on muscle preservation and nerve regeneration. In Int. Rev. Neurobiol. New York. 2009; PP: 445-64

32. Amoh $\mathrm{Y}$, Kanoh M, Niiyama S, Hamada $\mathrm{Y}$, Kawahara K, Sato $Y$, Hoffman RM, Katsuoka K. Human hair follicle pluripotent stem (hfPS) cells promote regeneration of peripheral-nerve injury: an advantageous alternative to ES and iPS cells. J. Cell. Biochem. 2009; 107(5); 1016-20.

33. Dezawa M, Takahashi I, Esaki M, Takano M, Sawada H. Sciatic nerve regeneration in rats induced by transplantation of in vitro differentiated bone marrow stromal cells. Eur. J. Neurosi. 2001;14; 1771-6.

34. Santiago LY, Clavijo- Alvarez J, Brayfield C, Rubin JP, Marra KG. Delivery of adipose derived precursors cells for peripheral nerve repair. Cell Tranplant. 2009; 18(2); 14558.

35. Kaspar BK, Llado J, Sherkat N, Rothstein JD, Gage FH. Retrograde viral delivery of IGF-1 prolongs survival in a mouse ALS model. Science, 2003; 8 (301); 839-42.

\section{Sponsors/ Grants:}

This work was supported, in part, by Muscular Dystrophy Association, USA, The Devora Eleonora Kirshman Fund and Norma and Alan Aufzein chair for research of Parkinson's Disease, Tel Aviv University.

\section{Corresponding author:}

Michal Dadon Nachum,FMRC; Rabin Medical Center, Beilinson Campus, Tel Aviv University., Petah Tikva, 49100, ISRAEL; Phone: 97239376277;Fax: 97239376181;Email: michald9@post.tau.ac.il 